\title{
Molecular Dynamics of POPC Phospholipid Bilayers through the Gel to Fluid Phase Transition: An Incoherent Quasi-Elastic Neutron Scattering Study
}

\author{
U. Wanderlingh, ${ }^{1}$ C. Branca, ${ }^{1}$ C. Crupi, ${ }^{1}$ V. Conti Nibali, ${ }^{2}$ G. La Rosa, ${ }^{3}$ S. Rifici, ${ }^{1}$ \\ J. Ollivier, ${ }^{4}$ and G. D'Angelo ${ }^{1}$ \\ ${ }^{1}$ Dipartimento di Scienze Matematiche e Informatiche, Scienze Fisiche e Scienze della Terra, Università di Messina, \\ Via F. Stagno d'Alcontres 31, 98166 Messina, Italy \\ ${ }^{2}$ Institute for Physical Chemistry II, Ruhr-University Bochum, Bochum, Germany \\ ${ }^{3}$ Physik-Department, Technische Universität München, Munich, Germany \\ ${ }^{4}$ Institut Laue-Langevin, 6 rue J. Horowitz, BP 156, 38042 Grenoble, France
}

Correspondence should be addressed to U. Wanderlingh; uwanderlingh@unime.it and G. D’Angelo; gdangelo@unime.it

Received 9 March 2017; Accepted 20 August 2017; Published 8 October 2017

Academic Editor: José L. Arias Mediano

Copyright (C) 2017 U. Wanderlingh et al. This is an open access article distributed under the Creative Commons Attribution License, which permits unrestricted use, distribution, and reproduction in any medium, provided the original work is properly cited.

\begin{abstract}
The microscopic dynamics for the gel and liquid-crystalline phase of highly aligned $\mathrm{D}_{2} \mathrm{O}$-hydrated bilayers of 1-palmitoyl-oleoylsn-glycero-phosphocholine (POPC) were investigated in the temperature range from 248 to $273 \mathrm{~K}$ by using incoherent quasielastic neutrons scattering (QENS). We develop a model for describing the molecular motions of the liquid phase occurring in the 0.3 to 350 ps time range. Accordingly, the complex dynamics of hydrogen are described in terms of simple dynamical processes involving different parts of the phospholipid chain. The analysis of the data evidences the existence of three different motions: the fast motion of hydrogen vibrating around the carbon atoms, the intermediate motion of carbon atoms in the acyl chains, and the slower translational motion of the entire phospholipid molecule. The influence of the temperature on these dynamical processes is investigated. In particular, by going from gel to liquid-crystalline phase, we reveal an increase of the segmental motion mainly affecting the terminal part of the acyl chains and a change of the diffusional dynamics from a localized rattling-like motion to a confined diffusion.
\end{abstract}

\section{Introduction}

The lipid bilayer is one of the most important self-assembled structures in nature. It regulates the flow of nutrients signaling events between cells and provides a selective barrier for cells and subcellular structures. It is also the structural host for functional proteins that control molecular traffic and cellular communication across the membrane. Membrane phospholipid bilayers are extensively used as model membranes for investigating the biological processes that occur at the cellular level [1]. In the last years, they received an increasing interest owing to a growing number of applications in biophysical and biomedical research, which include the passive transport through biomembranes [2], the pharmacokinetics of drugs $[3,4]$ or anaesthetics [5-8], and the lodging of membrane proteins [9]. Despite the efforts in studying these properties, many challenges still remain for the proper design of highly performing systems. To successfully apply the biomembrane technologies to real problems, it is necessary to deepen the understanding of the mechanisms that regulate the relative mobility (fluidity) of the individual lipid molecules in the bilayer [10] and to clarify how this mobility is influenced by changes in the environmental conditions (e.g., hydration and temperature) [11-13]. In particular, the fluidity of the membrane lipid bilayer is believed to play a main role in controlling the cell growth [14] and in modulating the function of transmembrane proteins and membrane enzymes [15], just to cite some notable examples.

As well known, lipid membranes have a rich phase diagram that is dominated by a main transition temperature, $T_{m}$, 
between the gel phase $\left(L_{\beta}\right)$ and the liquid-crystalline phase $\left(L_{\alpha}\right)$ [16]. For most phospholipids of practical interest, the phase transition temperature ranges from 270 to 310 Kelvin; it is higher for those phospholipids with longer tails, whereas the presence of unsaturated carbon bonds reduces $T_{m}$. The membrane fluidity critically depends on the structural phase state of lipids. In the gel phase, van der Waals interactions cause highly ordered packing of the acyl chains, which can also be tilted with respect to the lipid bilayer normal, resulting in limited rotational and translational motions. The gel phase is characterized by very low lipid mobility in the bilayer plane [17]. At the gel-liquid transition, the hydrocarbon chains change from an all-trans to a trans-gauche conformation. In the fluid phase, the lipid hydrocarbon chains experience higher translational and rotational mobility compared to the gel phase, resulting in enhanced membrane fluidity [18].

Several studies have demonstrated the coexistence of gel and liquid phases in living cell membranes [19] and in model membrane as well $[20,21]$. Other recent studies have highlighted the existence of a complex collective $\mathrm{THz}$ dynamics of phospholipid membrane $[22,23]$ and of an anomalous trend of the in-plane collective dynamics in crossing the main phase transition [24]. These anomalous vibrational dynamics were proven to be correlated with the formation of short-lived nanometer-scale lipid clusters that play a role in facilitating the passive molecular transport across the bilayer plane [24].

Consequently, studies addressing the polymorphic phase transitions of phospholipids, the dynamics and conformation of phospholipids in both gel and liquid-crystalline states, and the phase coexistence and phase interconversion in membrane model systems are of utmost importance to better understand the biological function of cell membranes.

In this regard, deeper knowledge of the single-particle dynamics of lipid molecules in bilayers above, at, and below the phase transition is highly desirable.

Recently, we have studied the liquid phase of hydrated DMPC and POPC bilayers [25] by means of quasi-elastic incoherent neutron scattering measurements and have identified three dominant dynamical processes on the ns-ps time scale, ascribed to three different motions of phospholipid molecules: (i) a fast uniaxial rotational diffusion, (ii) conformational, jump-like dynamics of the acyl chain along with threefold rotation of methyl groups, and (iii) overall diffusion dynamics confined within the first-neighbours cages. Here we present an extension of our previous investigation [25]. In this study, we examine the changes in the dynamics of hydrated POPC bilayers in the gel phase and near the gel-liquid phase transition temperature.

\section{Experimental Details and Data Analysis}

POPC is a synthetic monounsaturated phospholipid with a phosphocholine head group and one saturated 16:0 fatty acid chain and one unsaturated 18:1 fatty acid chain with two ester carbonyls (see Figure 1). Powder POPC was purchased from ROF Corporation.

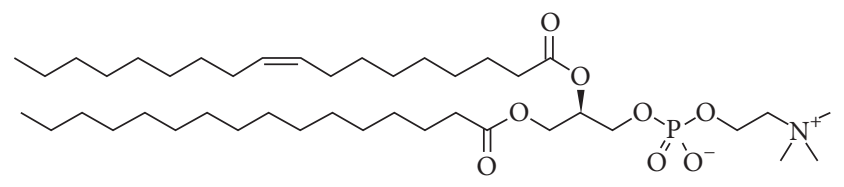

FIgure 1: Chemical structure of POPC.

Mica V-1 grade Muscovite $\mathrm{KAl}_{2}\left(\mathrm{Si}_{3} \mathrm{Al}\right) \mathrm{O}_{10}(\mathrm{OH}, \mathrm{F})_{2}$ sheets were used as substrate to support highly oriented and hydrated multilayer stacks.

The samples were hydrated with $28 \mathrm{D}_{2} \mathrm{O}$ molecules per lipid. Details of sample preparation are reported elsewhere [25].

QENS experiments were performed using the time-offlight spectrometer IN5 at Institut Laue-Langevin, Grenoble, France. The incident wavelength was $8 \AA$ and the Q-range covered $0.2-1.3 \AA^{-1}$ with an energy resolution of $10 \mu \mathrm{eV}$. All spectra were recorded for the $135^{\circ}$ orientation of planar membrane stacks relative to the incident beam at the temperatures of $248 \mathrm{~K}$ and $273 \mathrm{~K}$, respectively, below and close to the gelliquid phase transition temperature of POPC $\left(T_{m}=271 \mathrm{~K}\right)$.

The spectra were corrected for absorption and selfshielding and then converted to dynamic structure factor $S(\mathbf{Q}, \hbar \omega)$ and interpolated at constant $\mathbf{Q}$ values. Correction for multiple scattering was estimated to be negligible because the sample transmission was $\geq 90 \%$.

Quasi-elastic neutron scattering can provide valuable information about microscopic dynamics of atoms or molecules on time scale ranging from picoseconds to nanoseconds [26]. It is widely employed in the investigation of biological systems, including phospholipids bilayers [11, 27-29]. When applied to organic or biological systems, which are hydrogen-rich, neutron scattering spectra are dominated by the high incoherent cross section of $\mathrm{H}$ and essentially provide information on single-particle dynamics. In the case of phospholipids, hydrogen atoms are evenly distributed over the molecule and they can act as a marker for average phospholipid diffusion and lipid chain dynamics.

The incoherent scattering function $S_{\text {inc }}(\mathbf{Q}, \omega)$ in the elastic region is usually separated into an elastic "delta" component, $A_{0}(\mathbf{Q}) \delta(\omega)$, and a quasi-elastic component, $A_{1}(\mathbf{Q}) L(\omega)$, where $L(\omega)$ is a Lorentzian function, centered on $\omega=0$. The "delta" term and the Lorentzian term convey information on the geometry of the diffusion and on the time scale of diffusion, respectively. The fall in the elastic intensity as a function of $\mathbf{Q}$ is described by a form factor called the elastic incoherent structure factor (EISF), which is defined as

$$
\text { EISF }=\frac{A_{0}(\overrightarrow{\mathbf{Q}})}{A_{0}(\overrightarrow{\mathbf{Q}})+A_{1}(\overrightarrow{\mathbf{Q}})}=\frac{\text { elastic intensity }}{\text { total intensity }}
$$

The exact shape of EISF is indicative of the geometry of the diffusional process and, by a careful line shape analysis of the spectra, it is possible to distinguish different dynamics that can be correlated to specific molecular groups in the system.

The bound cross sections for the whole POPC molecule for the component groups and for $28 \mathrm{D}_{2} \mathrm{O}$ molecules were 
TABLE 1: Total cross section (barn) for a single phospholipid (POPC) with 28 hydration water molecules. Percentage values for constituent groups are given relative to the total cross section for a whole phospholipid.

\begin{tabular}{|c|c|c|c|c|}
\hline \multirow{3}{*}{$\begin{array}{l}\text { Water } \\
\text { Phospholipid }\end{array}$} & \multirow{3}{*}{$\begin{array}{r}28 \mathrm{D}_{2} \mathrm{O} \\
\text { POPC }\end{array}$} & $\sigma_{\text {inc }}$ & $\sigma_{\text {coh }}$ & $\% \sigma$ \\
\hline & & 114.8 & 431.7 & 7.2 \\
\hline & & 6581.8 & 421. & 92.8 \\
\hline Head methyls & $3 \mathrm{CH}_{3}$ & 722.3 & 32.5 & 10.8 \\
\hline Head linkers & $4 \mathrm{CH}_{2}+\mathrm{CH}+2 \mathrm{C}$ & 722.3 & 54.7 & 11.1 \\
\hline Head phosphate & $\mathrm{N}+8 \mathrm{O}+\mathrm{P}$ & 0.5 & 48.2 & 0.7 \\
\hline Tail acyl chains & $29 \mathrm{CH}_{2}$ & 4655.1 & 265.4 & 70.2 \\
\hline Tail methyls & $2 \mathrm{CH}_{3}$ & 481.6 & 21.6 & 7.2 \\
\hline
\end{tabular}

calculated and listed in Table 1. To a good approximation, the $\mathrm{D}_{2} \mathrm{O}$-hydrated sample exhibits only scattering from the phospholipids. In fact, the incoherent scattering cross section for a POPC molecule is about $95 \%$ of the total cross section and is due to the predominant incoherent scattering of hydrogen in the biomolecules. Nevertheless, water contribution was explicitly taken into account as described in the supplementary material of our previous study [25].

\section{Results and Discussion}

Similar to what was observed for the liquid phase [25] and in general for all biological macromolecules, the QENS spectral line of the gel phase of POPC/ $\mathrm{D}_{2} \mathrm{O}$ system appears to be broadened over a very wide energy range with respect to the resolution function (see Figure 2). This feature is a signature of the existence of structural fluctuations on the picosecond time scale. The notable extension of the broadening implies that a single Lorentzian cannot accurately model the spectrum and suggests that more than one dynamic contributes to the quasi-elastic scattering. Thus we analyzed the neutron data following the same procedure and applying the same model used for the POPC liquid phase [25].

The incoherent contribution from the phospholipid dynamics, $S_{\text {lip }}(\mathbf{Q}, \hbar \omega)$, was calculated as the sum of a delta function $(\delta(\hbar \omega))$ and a set of Lorentzians [11, 30, 31]:

$$
S_{\text {lip }}(\mathbf{Q}, \hbar \omega)=A_{0} \delta(\hbar \omega)+\sum_{i=1}^{3} \frac{A_{i}}{\pi} \frac{\Gamma_{i}}{\Gamma_{i}^{2}+\hbar \omega^{2}} .
$$

The delta function represents the elastic response, while the Lorentzian contributions account for the relaxational motions that cause the quasi-elastic broadening of the elastic peak. The HWHMs (Half Widths at Half Maximums) of the Lorentzian contributions, $\Gamma_{i}$, are directly related to the characteristic times associated with the motions of the scattering nuclei involved: $\tau_{i}=\hbar / \Gamma_{i}$.

Notably, as for the liquid phase [25], three Lorentzians were required to adequately fit the gel phase of POPC bilayers. An example of the quality of the fit of the gel phase of POPC $(T=248 \mathrm{~K})$ at $\mathbf{Q}=0.7 \AA^{-1}$ is shown in Figure 2. The fitting procedure shows/suggests the presence of a very broad contribution of about $1800-2500 \mu \mathrm{eV}$, a second contribution of about $80-120 \mu \mathrm{eV}$, and a narrow contribution ranging from 5 to $15 \mu \mathrm{eV}$. As already observed in the liquid phase, the values
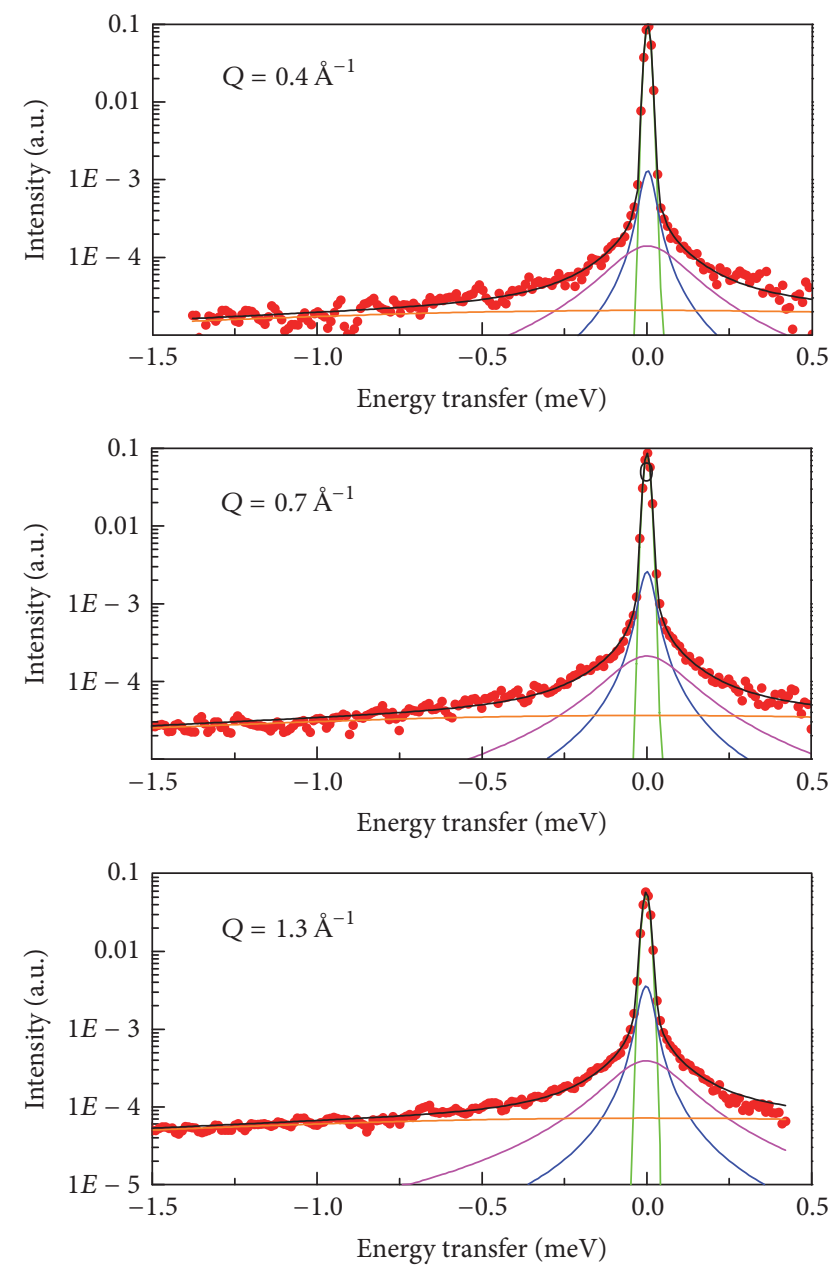

FIgUre 2: Semilog plot of the QENS intensity for POPC at $248 \mathrm{~K}$ at three different $\mathbf{Q}$ values. The best fit of equation (2) is displayed as a black continuous line.

of $\Gamma_{i}$ differ nearly by an order of magnitude over the entire Q-range (data not shown). More precisely, these findings confirm the existence of three dominant dynamical processes taking place on different time scales in both structural phases.

To properly describe each of these dynamics and analyze the effect of temperature on the conformational motions of lipid bilayers, the same microscopic model proposed to 


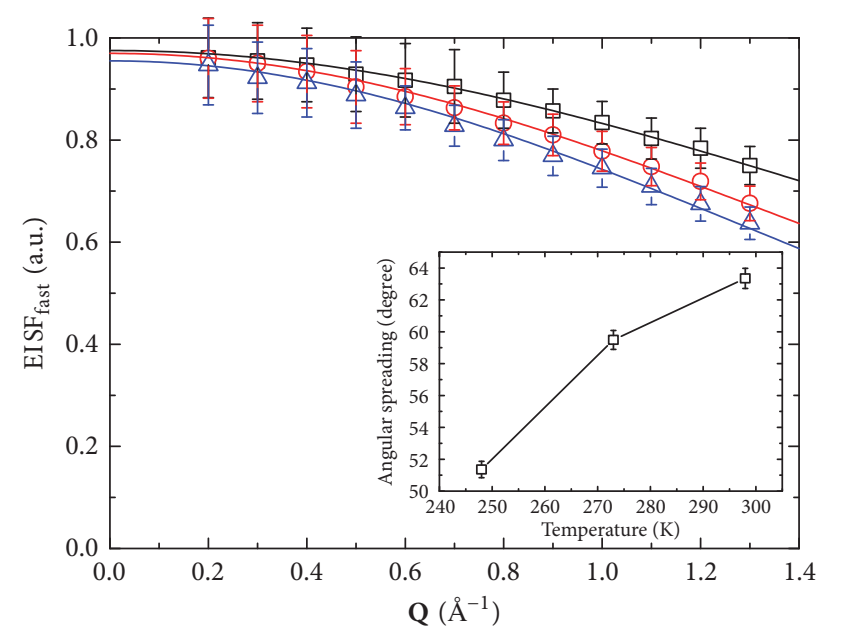

$\square T=248 \mathrm{~K}$

○ $T=273 \mathrm{~K}$

$\triangle T=298 \mathrm{~K}$

FIGURE 3: Q-dependence of the experimental EISF $_{\text {fast }}$ at $T=248 \mathrm{~K}$ and $273 \mathrm{~K}$ compared with the data at $T=298 \mathrm{~K}$ taken from [25]. Solid lines are fits to the uniaxial rotation model. In the inset, the temperature dependence of the angular spreads is shown.

describe the hydrogen dynamics for the liquid phase of phospholipid bilayers was considered.

In this model, the dynamical structure factor $S(\mathbf{Q}, \hbar \omega)$ is described by a convolution of three types of motions (see [25] and supplementary materials therein for more details). Each contribution describes the dynamics for the protons in a specific confined geometry and is represented as the sum of an EISF and an elastic term. The three dynamics evolve on three different time scales: fast, intermediate, and slow.

The fast process occurs on the picoseconds time scale ( $\hbar / \Gamma \approx 0.33 \mathrm{ps}$ ) and is believed to describe the hydrogen motion with respect to the acyl carbon atoms that act like a center of mass for a restricted librational motion about the $\mathrm{C}-\mathrm{H}$ bond direction [25]. Similar to what was observed for liquid crystals $[32,33]$, this motion can be described by the uniaxial rotational diffusion model [34]. The corresponding EISF, averaged over all the possible orientations of the bond direction with respect to $\mathbf{Q}$, can be written as follows:

$$
\operatorname{EISF}_{\text {fast }}(\mathbf{Q})=\sum_{l=0}^{\infty}(2 l+1) j_{l}^{2}(\mathbf{Q} R) S_{l}^{2}(\mu),
$$

where $\mathrm{R}$ is the $\mathrm{C}-\mathrm{H}$ bond length and $S_{l}(\mu)$ is an orientational order parameter dependent on the width of the angular distribution, $\mu$, which is the only fitting parameter [25].

The experimental EISF for this component is shown in Figure 3 for POPC at the two investigated temperatures, $T=$ 248 and $273 \mathrm{~K}$, and compared with the results obtained at $T=298 \mathrm{~K}$ for the liquid phase [25]. The best-fit curves are also shown. As can be inferred from the figure, the model satisfactorily reproduces the experimental data, suggesting that the fast, small amplitude fluctuation of the bond angle between $\mathrm{H}$ and $\mathrm{C}$ atoms in the $\mathrm{CH}_{2}$ groups is responsible for these dynamics. Nevertheless, the possibility that a similar

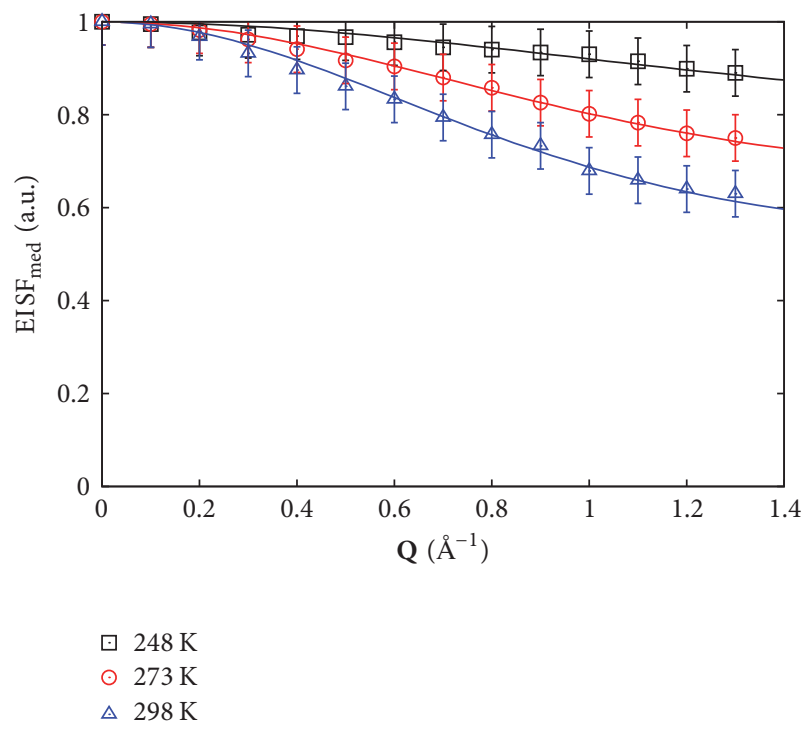

FIGURE 4: Q-dependence of the experimental EISF $_{\text {med }}$ for POPC at three temperatures. The solid lines are fits obtained with the bead model, described in the text.

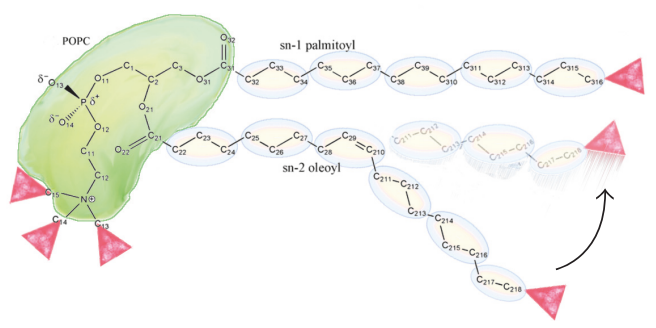

FIGURE 5: Sketch of the bead model for POPC. See text for details.

hindered libration of the bond angle takes place also in the methyl groups cannot be a priori excluded.

The values of angular spreads, $\Delta \alpha=\cos ^{-1}\left(S_{l}(\mu)\right)$, obtained from the fitting procedure, are shown in the inset of Figure 3 as a function of temperature. The increase of $\Delta \alpha$ value with temperature describes wider spreading of the hydrogen position and can be explained due to the softening of the interchain interactions occurring during the passage from the gel to the liquid-crystalline phase.

The intermediate dynamics are characterized by a time $(\tau=\hbar / \Gamma)$ of $6-7 \mathrm{ps}$, which is the typical time of both the conformational dynamics in the lipid chains and the rotational diffusion of methyl groups $[11,35,36]$. In Figure 4, the experimental EISF $_{\text {med }}$, derived by the line shape analysis, for the POPC at $T=248$ and $273 \mathrm{~K}$, are shown and compared with the results obtained at $T=298 \mathrm{~K}$ for the liquid phase. These data were fitted by means of a simple "bead model": the phospholipid molecule was built up with several linked beads representing head groups, chain segments, head methyls, and tail methyls (see Figure 5).

In this scheme, the intermediate time scale motion is described in terms of two processes: a two-site jump transition between trans and gauche conformation of carbon atoms in the $\mathrm{CH}_{2}$ groups and a rotation of the $\mathrm{CH}_{3}$ methyl groups 
[25]. It is worth noting that in the present paper the "bead model" was improved by increasing the number of beads modelling the chains. Increasing the number of beads results in the increase of spatial resolution and in the possibility to consider in a more realistic way the chain dynamics of POPC, which shows a rigid cis double bond in the middle of one of the two hydrocarbon chains.

More specifically, the first bead, termed as 0 , accounts for the $\mathrm{CH}_{2}$ groups in the polar head. The other beads, six for the unsaturated and longer (oleoyl) chain and five for the saturated (palmitoyl) chain, account for the segmental dynamics of the chain. Each bead includes two single $\mathrm{C}-\mathrm{C}$ bonds and can perform a two-site jump motion. Of course each bead displacement is convoluted with that of the preceding beads. The rotational motions of the head and terminal methyl groups, also modelled as small beads, are included in the model.

For the two-site jump dynamics, EISF is given as follows [37]: $\operatorname{EISF}_{i}(\mathbf{Q})=1-2 P 1 P 2\left(1-j_{0}(\mathbf{Q} d)\right)$, where $P 1$ and $P 2$ are the probabilities of finding a bead in a trans or gauche conformation, respectively, with $(P 1+P 2)=1$. The value of the jump length was chosen to be equal to the distance between the carbon positions in the two conformations: $d=$ $2.49 \AA$.

As far as the methyl group dynamics are concerned, they are described by a three-site jump rotational diffusion model, and the corresponding EISF is given by [26]

$$
\mathrm{EISF}_{m}=\frac{\left(1+J_{0}\left(\mathbf{Q} R_{m} \sqrt{3}\right)\right)}{3} .
$$

The contributions of the two dynamics were conveniently weighted taking into account the sample composition. Furthermore, since the motion of each segment has to be convoluted with the motion of the previous one, the dynamic structure factor (relative to a center of mass, corresponding to the head-tail contact) was obtained as the sum of products of the single contributions. Consequently, the resulting EISF $_{\text {bead }}$ for our model has been written as

$$
\begin{aligned}
\text { EISF }_{\text {bead }}= & A \text { Eisf }_{\text {meth }} \text { Eisf }_{0}+B \text { Eisf }_{0}+\frac{C_{u}}{6} \sum_{i=1}^{6} \prod_{j=1}^{i} \text { Eisf }_{i} \\
& +\operatorname{DEisf}_{6} \text { Eisf }_{\text {meth }}+\frac{C_{s}}{5} \sum_{i=1}^{5} \prod_{j=1}^{i} \text { Eisf }_{i} \\
& +\operatorname{DEisf}_{5} \text { Eisf }_{\text {meth }},
\end{aligned}
$$

where $A, B, C$, and $D$ are the fractional cross sections for the $\mathrm{CH}_{3}$ groups in the polar head, the $\mathrm{CH}_{2}$ groups in the polar head, the $\mathrm{CH}_{2}$ groups in the chains, and the terminal $\mathrm{CH}_{3}$ groups, respectively. Note that $C_{u}$ and $C_{s}$ indicate the contribution for the unsaturated and saturated chains. The corresponding fitted curves are shown in Figure 4 and the best-fit parameters of the probability $P 1_{i}$ for each bead are given in Table 2, where they are compared with the data obtained for the POPC liquid phase.

It is worth noting that the quality of the fit for the POPC liquid phase obtained by increasing the number of beads of
TABLE 2: Values for the probability of finding a bead in the lower energy site.

\begin{tabular}{lccc}
\hline & $248 \mathrm{~K}$ & $273 \mathrm{~K}$ & $298 \mathrm{~K}$ \\
\hline$P 1_{0}$ & 1.00 & 1.00 & 1.00 \\
$P 1_{1}$ & 1.00 & 1.00 & 1.00 \\
$P 1_{2}$ & 1.00 & 1.00 & 1.00 \\
$P 1_{3}$ & 1.00 & 1.00 & 0.93 \\
$P 1_{4}$ & 0.99 & 0.95 & 0.67 \\
$P 1_{5}$ & 0.98 & 0.77 & 0.49 \\
$P 1_{6}$ & 0.91 & 0.49 & 0.50 \\
\hline
\end{tabular}

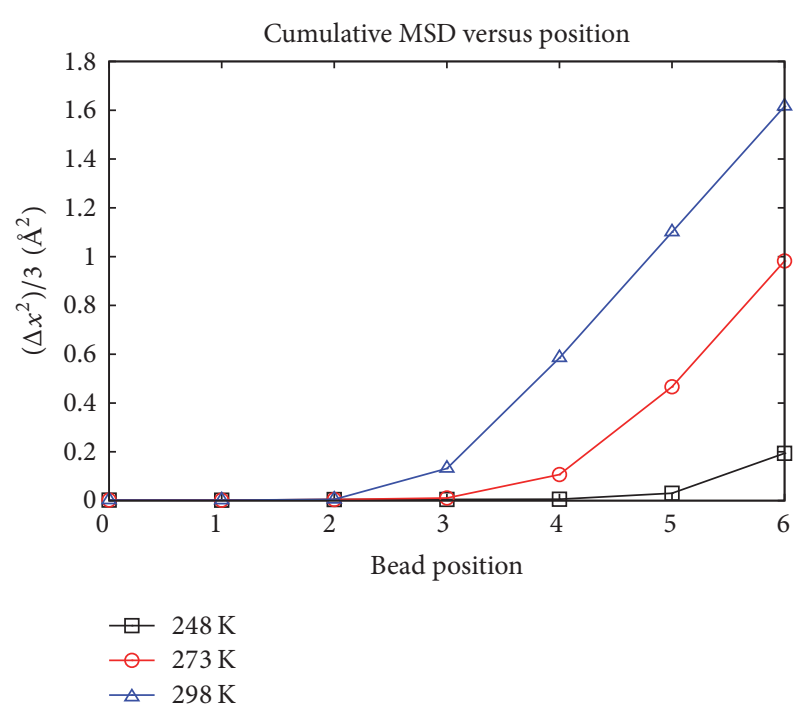

FIGURE 6: Mean square displacement as function of bead position for POPC at the investigated temperatures.

the model to seven was improved compared to the previous analysis [25]. Furthermore, we observe that the values of $P 1$ decrease nonlinearly as the position of carbon atoms in alkyl chain increases, showing a substantial reduction starting from the fourth bead. This indicates higher motional freedom of the acyl chain in the region below the doublebond position of the unsaturated chain of POPC. This finding suggests that the conformational sampling of the molecules is correctly represented in the level of resolution of the sevenbead model.

The ability of this model to describe the experimental data of both liquid and gel phases of phospholipid bilayers proves that the transition between trans and gauche conformation is a relevant motion for the lipid chain dynamics.

Within the used model, it is possible to evaluate the mean square displacement (MSD) from the jump probabilities $P 1$ and $P 2:\left\langle\Delta x^{2}\right\rangle=P 1 P 2 d^{2} / 3$. In Figure 6 , a plot of $\left\langle\Delta x^{2}\right\rangle$ as a function of bead position is shown. We observed that in the gel phase a residual motion associated with anti-gauche transition is only observed in correspondence of the terminal part of the chain. Conversely, rising the temperature, these dynamics become even more relevant and involve about half of chain at $T_{m}$, engaging up to $2 / 3$ of the chain when bilayers are in the liquid phase. The results for the jump length/rates 


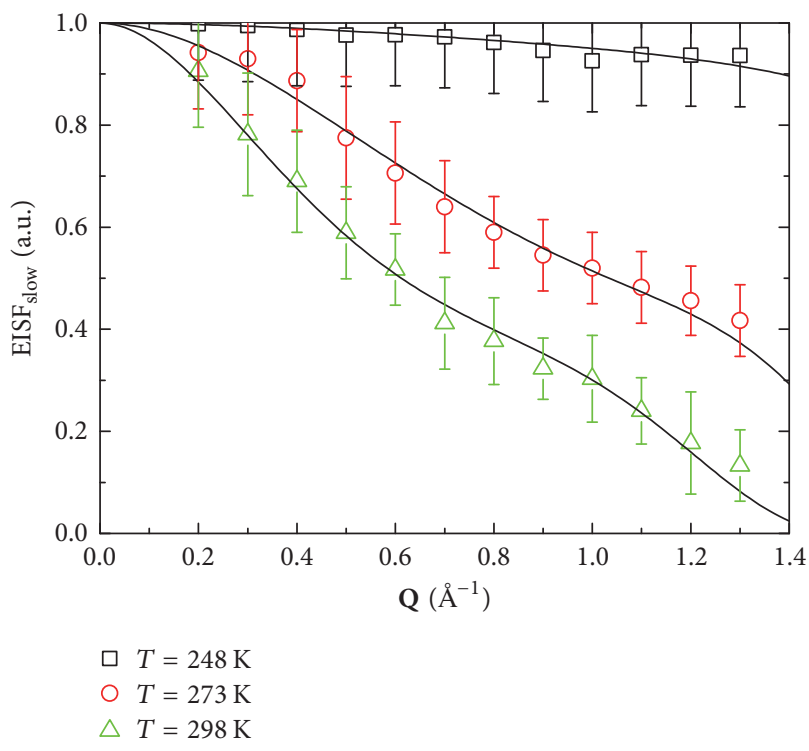

(a)

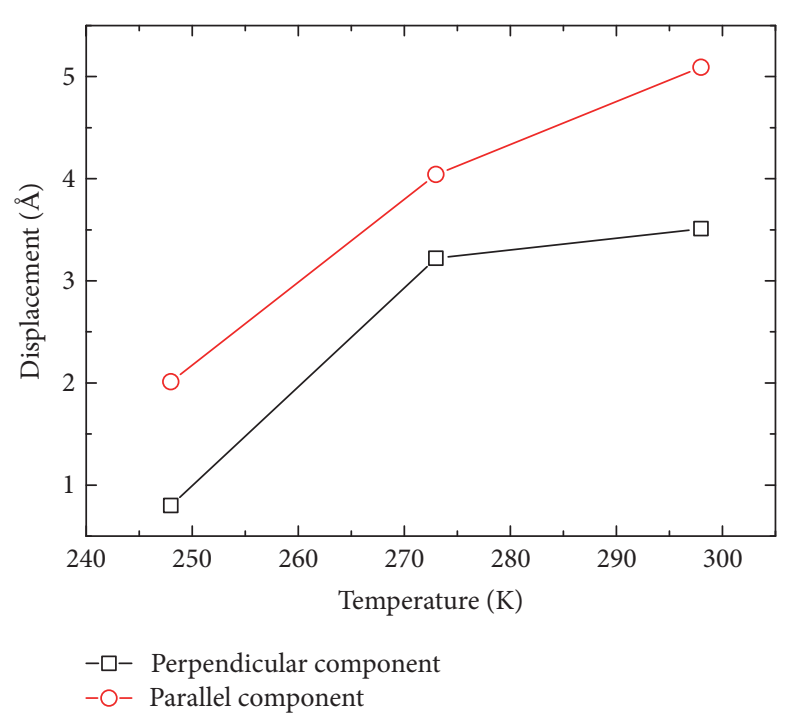

(b)

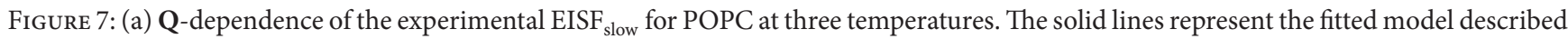
in the text (see equation (6)). (b) Parallel (in-plane) displacement and perpendicular (off-plane) displacement versus temperature.

and the MSD are consistent with those previously obtained on similar systems by neutron scattering techniques [31, 38, 39], even if the details of the chain motion are modelled by different approaches. The nonlinear increase of the motion's amplitude when moving down the chain can be associated with the order parameter obtained by NMR measurements in similar systems [40, 41].

Finally, we have examined the EISFs relative to the slow dynamics of the POPC gel phase $(\hbar / \Gamma$ ranging from 40 to $350 \mathrm{ps}$ ). Analogously to what was reported for the liquid phase [25], we assume that the slow component is associated with the translational mobility of the whole phospholipid and we describe it as a center-of-mass motion.

As first step, we adopted the same model used for the liquid phase [25], that is, a continuum diffusion inside a cylindrical shape. Anyway, this model resulted to be unsuitable to describe the experimental EISF at and below the main transition temperature (data not shown).

Conversely, we obtained a fairly good description using a simpler model similar to that described by (4) and based on the convolution of two jump diffusions for the in-plane and off-plane displacements, respectively. Within this framework, assuming that, in long times, a two-state equilibrium with $P 1$ equal to $P 2$ is reached, EISF of the slow motion can be written as follows:

$$
\begin{aligned}
& \operatorname{EISF}_{\text {slow }}(\mathbf{Q}) \\
& \quad=\frac{1}{4}\left(1+j_{0}\left(\overrightarrow{\mathbf{Q}} \cdot \vec{d}_{\|}\right)\right)\left(1+j_{0}\left(\overrightarrow{\mathbf{Q}} \cdot \vec{d}_{\perp}\right)\right) .
\end{aligned}
$$

Here the fitting parameters $d_{\|}$and $d_{\perp}$ are the jump distances for the in-plane and off-plane displacements, whereas the angle between the exchanged wave-vector $\vec{Q}$ and the displacement $\vec{d}$ is defined by the detector positions and the sample orientation.

In Figure 7(a) the experimental EISF and the fitting curves for the phase at and below the main transition are reported and compared with the results for the liquid phase obtained by the continuous diffusion model inside a cylinder [25]. In Figure 7(b) the best-fit values for $d_{\|}$ and $d_{\perp}$ as a function of temperature are shown. Note that the displacements for the liquid phase reported here are the diameter and the height of the confining cylindrical region.

It is worth observing that the structure factor increases as the temperature is decreased, indicating that by going from the liquid to the gel phase the motion becomes more and more restricted. Furthermore, while the in-plane displacement exhibits a nearly linear increase with temperature, the perpendicular component shows saturation. This is consistent with the fact that the transition from the liquid into the gel phase involves a drastic decrease in volume and area of the lipid molecules, which results in significant strengthening of the van der Waals attractive forces in the lipid bilayer. The increase in the packing density of acyl chains imposes geometrical constraints that restrict the local diffusion of lipid molecules, affecting mainly the out-of-plane direction. It is also reasonable to consider the observed saturation as a consequence of the limited protrusion allowed for phospholipids packed in a bilayer. We infer that the inadequacy of the model of diffusion into a cylinder in describing the slow dynamics at and below the main transition should indicate that the phospholipid diffusion in the gel phase is rattling-like with short jumps around a quasi-equilibrium position [42], while in the liquid phase the dynamics, although rather confined, evolve into a continuous diffusion. However, because of the limited instrumental resolution, we are not able to identify 
with certainty a diffusive motion outside the confinement region for both liquid and gel phases.

\section{Conclusion}

The microscopic dynamics of lipid multibilayer POPC system in the gel phase and at the gel-liquid phase transition were investigated by means of incoherent quasi-elastic neutron scattering and compared with the dynamics previously observed in the liquid phase. In both phases, the line shape analysis of the QENS spectra revealed the presence of three contributions to the global dynamics of phospholipid. Such contributions take place on well-separated time scales and were ascribed to different parts of the phospholipids, that is, local hydrogen libration, segmental acyl tails motion, and overall diffusion. The present analysis is based on the refinement of a model, recently developed to describe the ps time scales dynamics for the liquid phase of bilayers. This model was revealed to be more accurate and suitable for describing the dynamics of more complex phospholipids characterized by unsaturated and asymmetric chains.

Through the analysis of the temperature dependence of EISF, it was possible to infer that the gel to liquid phase transition entails (i) softening of the interchains interaction, (ii) an increase of the segmental motion occurring in the final part of the acyl chain, and (iii) a change of the diffusional dynamics from a very localized rattling-like motion to a confined diffusion.

This study provides valuable data for characterizing the dynamical behaviour of lipid bilayers. The simplicity of the model allows for its application in studying various subjects such as lipid raft and more complicated cases where functional components are included in the membrane bilayer.

\section{Conflicts of Interest}

The authors declare that there are no conflicts of interest regarding the publication of this paper.

\section{References}

[1] T. Yang, O. K. Baryshnikova, H. Mao, M. A. Holden, and P. S. Cremer, "Investigations of bivalent antibody binding on fluid-supported phospholipid membranes: The effect of hapten density," Journal of the American Chemical Society, vol. 125, no. 16, pp. 4779-4784, 2003.

[2] S. Paula, A. G. Volkov, A. N. Van Hoek, T. H. Haines, and D. W. Deamer, "Permeation of protons, potassium ions, and small polar molecules through phospholipid bilayers as a function of membrane thickness," Biophysical Journal, vol. 70, no. 1, pp. 339348, 1996.

[3] J. L. Arias, "Liposomes in drug delivery: a patent review (2007 present)," Expert Opinion on Therapeutic Patents, vol. 23, pp. 1399-1414, 2013, Arias, J. L. Expert Opin. Ther. Pat.2013,23, 13991414.

[4] M. A. Kiselev and D. Lombardo, "Structural characterization in mixed lipid membrane systems by neutron and X-ray scattering," Biochimica et Biophysica Acta - General Subjects, vol. 1861, no. 1, pp. 3700-3717, 2017.
[5] U. Wanderlingh, G. D’Angelo, V. Conti Nibali et al., "Interaction of alcohol with phospholipid membrane: NMR and XRD investigations on DPPC-hexanol system," Spectroscopy, vol. 24, no. 3-4, pp. 375-380, 2010.

[6] S. Rifici, C. Corsaro, C. Crupi et al., "Lipid diffusion in alcoholic environment," Journal of Physical Chemistry B, vol. 118, no. 31, pp. 9349-9355, 2014.

[7] S. Rifici, G. D’Angelo, C. Crupi et al., "Influence of alcohols on the lateral diffusion in phospholipid membranes," Journal of Physical Chemistry B, vol. 120, no. 7, pp. 1285-1290, 2016.

[8] H. A. Pillman and G. J. Blanchard, "Effects of ethanol on the organization of phosphocholine lipid bilayers," Journal of Physical Chemistry B, vol. 114, no. 11, pp. 3840-3846, 2010.

[9] G. D’angelo, U. Wanderlingh, V. C. Nibali, C. Crupi, C. Corsaro, and G. Di Marco, "Physical study of dynamics in fully hydrated phospholipid bilayers," Philosophical Magazine, vol. 88, no. 3335, pp. 4033-4046, 2008.

[10] H. K. Kimelberg, "Alterations in phospholipid-dependent $(\mathrm{Na}++\mathrm{K}+)$-ATPase activity due to lipid fluidity. Effects of cholesterol and Mg2+," BBA - Biomembranes, vol. 413, no. 1, pp. 143-156, 1975.

[11] J. Fitter, R. Lechner, G. Buldt, and N. Dencher, "Internal molecular motions of bacteriorhodopsin: hydration-induced flexibility studied by quasielastic incoherent neutron scattering using oriented purple membranes," National Academy of Sciences of the United States of America, vol. 93, pp. 7600-7605, 1996.

[12] J. Fitter, R. Lechner, and N. Dencher, "Interactions of hydration water and biological membranes studied by neutron scattering," The Journal of Physical Chemistry B, vol. 103, pp. 8036-8050, 1999.

[13] S. Rifici, C. Crupi, G. D’Angelo et al., "Effects of a short length alcohol on the dimyristoylphosphatidylcholine system," Philosophical Magazine, vol. 91, no. 13-15, pp. 2014-2020, 2011.

[14] R. McElhaney, Biomembranes, vol. 12, Plenum Press, New York, NY, USA, 1984.

[15] R. McElhaney, Current Topics in Membranes and Transport, vol. 17, Academic Press, New York, NY, USA, 1982.

[16] J. F. Nagle and S. Tristram-Nagle, "Structure of lipid bilayers," Biochimica et Biophysica Acta-Reviews on Biomembranes, vol. 1469, no. 3, pp. 159-195, 2000.

[17] R. N. A. H. Lewis and R. N. McElhaney, The Structure of Biological Membranes, vol. 2, CRC press, 3rd edition, 2005.

[18] G. Pabst, H. Amenitsch, D. P. Kharakoz, P. Laggner, and M. Rappolt, "Structure and fluctuations of phosphatidylcholines in the vicinity of the main phase transition," Physical Review E - Statistical, Nonlinear, and Soft Matter Physics, vol. 70, no. 2, Article ID 021908, pp. 1-21908, 2004.

[19] G. van Meer, D. R. Voelker, and G. W. Feigenson, "Membrane lipids: where they are and how they behave," Nature Reviews Molecular Cell Biology, vol. 9, no. 2, pp. 112-124, 2008.

[20] A. F. Xie, R. Yamada, A. A. Gewirth, and S. Granick, "Materials Science of the Gel to Fluid Phase Transition in a Supported Phospholipid Bilayer," Physical Review Letters, vol. 89, no. 24, Article ID 246103, 2002.

[21] M. C. Rheinstädter, C. Ollinger, G. Fragneto, F. Demmel, and T. Salditt, "Collective dynamics of lipid membranes studied by inelastic neutron scattering," Physical Review Letters, vol. 93, no. 10, pp. 1-108107, 2004.

[22] V. C. Nibali, G. D’Angelo, and M. Tarek, "Molecular dynamics simulation of short-wavelength collective dynamics of phospholipid membranes," Physical Review E, vol. 89, no. 5, Article ID 050301, 2014. 
[23] D. Angelo, G. C. Nibali, V. Crupi et al., "Probing intermolecular interactions in phospholipid bilayers by far-infrared spectroscopy," The Journal of Physical Chemistry B, vol. 121, no. 6, pp. 1204-1210, 2017.

[24] M. Zhernenkov, D. Bolmatov, D. Soloviov et al., "Revealing the mechanism of passive transport in lipid bilayers via phononmediated nanometre-scale density fluctuations," Nature Communications, vol. 7, Article ID 11575, 2016.

[25] U. Wanderlingh, G. D’Angelo, C. Branca et al., "Multicomponent modeling of quasielastic neutron scattering from phospholipid membranes," Journal of Chemical Physics, vol. 140, no. 17, Article ID 174901, 2014.

[26] M. Bee, Quasielastic Neutron Scattering, Adam Hilger, Bristol, 1988.

[27] W. Pfeiffer, T. H. Henkel, E. Sackmann, W. Knoll, and W. Knoll, "Local dynamics of lipid bilayers studied by incoherent quasielastic neutron scattering," EPL, vol. 8, no. 2, pp. 201-206, 1989.

[28] E. Endress, H. Heller, H. Casalta, M. F. Brown, and T. M. Bayerl, "Anisotropic motion and molecular dynamics of cholesterol, lanosterol, and ergosterol in lecithin bilayers studied by quasielastic neutron scattering," Biochemistry, vol. 41, no. 43, pp. 13078-13086, 2002.

[29] G. Schiro, M. Sclafani, C. Caronna, F. Natali, M. Plazanet, and A. Cupane, "Dynamics of myoglobin in confinement: an elastic and quasi-elastic neutron scattering study," Chemical Physics, vol. 345, no. 2-3, pp. 259-266, 2008.

[30] S. König, W. Pfeiffer, T. Bayerl, D. Richter, and E. J. Sackmann, Journal de Physique II, vol. 2, pp. 1589-1615, 1992.

[31] Y. Gerelli, V. G. Sakai, J. Ollivier, and A. Deriu, "Conformational and segmental dynamics in lipid-based vesicles," Soft Matter, vol. 7, no. 8, pp. 3929-3935, 2011.

[32] F. Volino, A. J. Dianoux, and H. Hervet, "Neutron quasi-elastic scattering study of rotational motions in the smectic $\mathrm{C}, \mathrm{H}$ and VI phases of terephtal-bis-butyl-aniline (TBBA)," Le Journal de Physique Colloques, vol. 37, no. C3, pp. C3-55-C3-64, 1976.

[33] A. J. Dianoux, F. Volino, and H. Hervet, "Incoherent scattering law for neutron quasi-elastic scattering in liquid crystals," Molecular Physics, vol. 30, no. 4, pp. 1181-1194, 1975.

[34] M. Bée, "Localized and long-range diffusion in condensed matter: State of the art of QENS studies and future prospects," Chemical Physics, vol. 292, no. 2-3, pp. 121-141, 2003.

[35] T. Pradeep, S. Mitra, A. Sreekumaran Nair, and R. Mukhopadhyay, "Dynamics of alkyl chains in monolayer-protected au and ag clusters and silver thiolates: A comprehensive quasielastic neutron scattering investigation," Journal of Physical Chemistry B, vol. 108, no. 22, pp. 7012-7020, 2004.

[36] S. König and E. Sackmann, "Molecular and collective dynamics of lipid bilayers," Current Opinion in Colloid and Interface Science, vol. 1, no. 1, pp. 78-82, 1996.

[37] W. Doster, S. Cusack, and W. Petry, "Dynamical transition of myoglobin revealed by inelastic neutron scattering," Nature, vol. 337, no. 6209, pp. 754-756, 1989.

[38] V. K. Sharma, E. Mamontov, D. B. Anunciado, H. Oneill, and V. Urban, "Nanoscopic dynamics of phospholipid in unilamellar vesicles: Effect of gel to fluid phase transition," Journal of Physical Chemistry B, vol. 119, no. 12, pp. 4460-4470, 2015.

[39] C. Castellano, F. Natali, D. Pozzi, G. Caracciolo, and A. Congiu, "Dynamical properties of oriented lipid membranes studied by elastic incoherent neutron scattering," Physica B: Condensed Matter, vol. 350, no. 1-3, pp. e955-e958, 2004.
[40] M. Lafleur, B. Fine, E. Sternin, P. Cullis, and M. Bloom, "Smoothed orientational order profile of lipid bilayers by $2 \mathrm{H}$ nuclear magnetic resonance," Biophysical Journal, vol. 56, pp. 1037-1041, 1989.

[41] L. S. Vermeer, B. L. De Groot, V. Réat, A. Milon, and J. Czaplicki, "Acyl chain order parameter profiles in phospholipid bilayers: computation from molecular dynamics simulations and comparison with $2 \mathrm{H}$ NMR experiments," European Biophysics Journal, vol. 36, no. 8, pp. 919-931, 2007.

[42] C. L. Armstrong, M. Trapp, J. Peters, T. Seydel, and M. C. Rheinstädter, "Short range ballistic motion in fluid lipid bilayers studied by quasi-elastic neutron scattering," Soft Matter, vol. 7, no. 18 , pp. 8358-8362, 2011. 

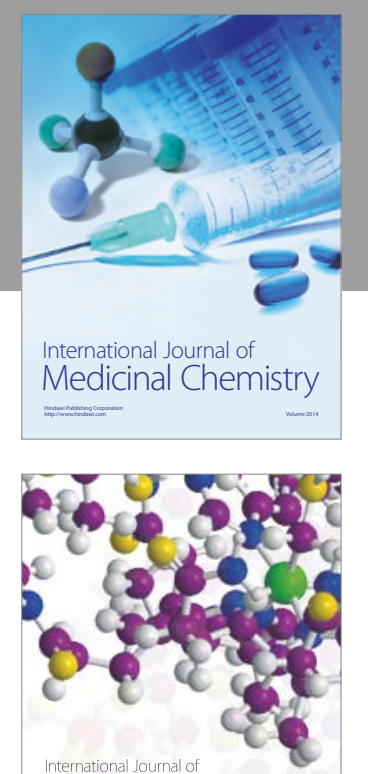

Carbohydrate Chemistry

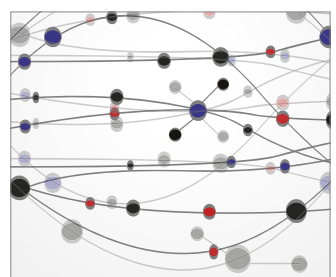

The Scientific World Journal
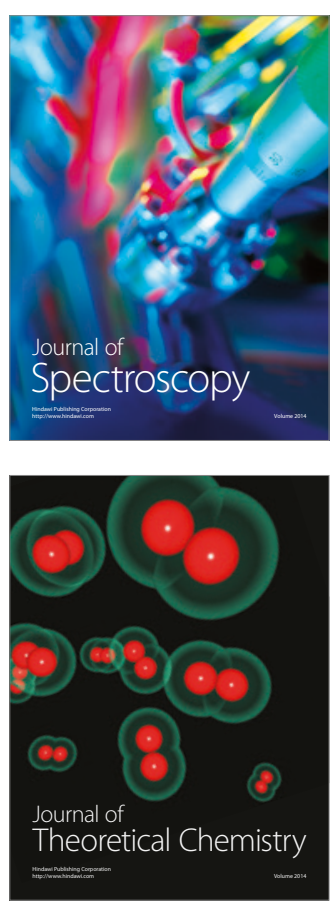
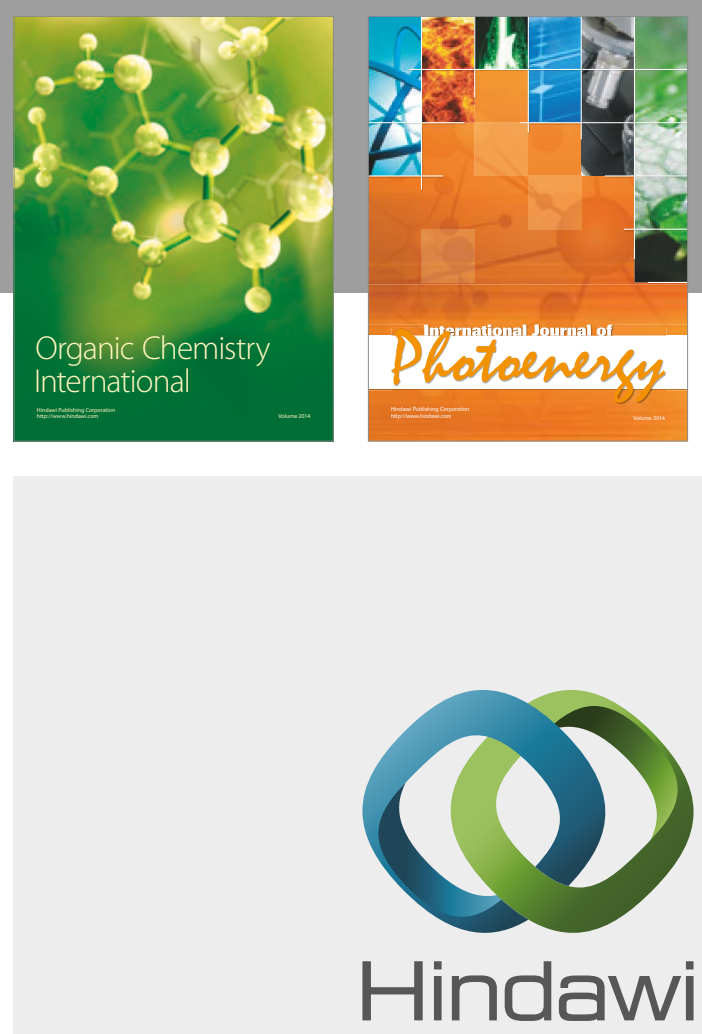

Submit your manuscripts at

https://www.hindawi.com

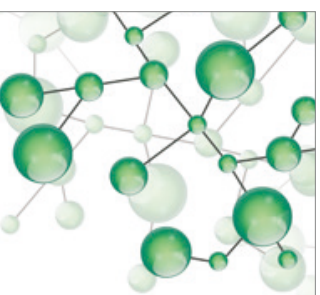

International Journal of

Inorganic Chemistry

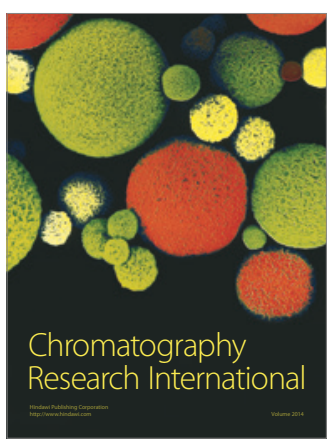

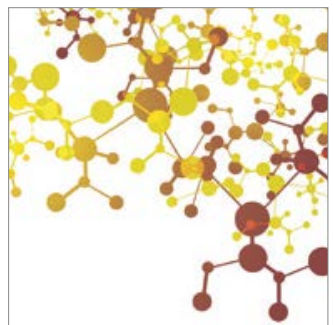

Applied Chemistry
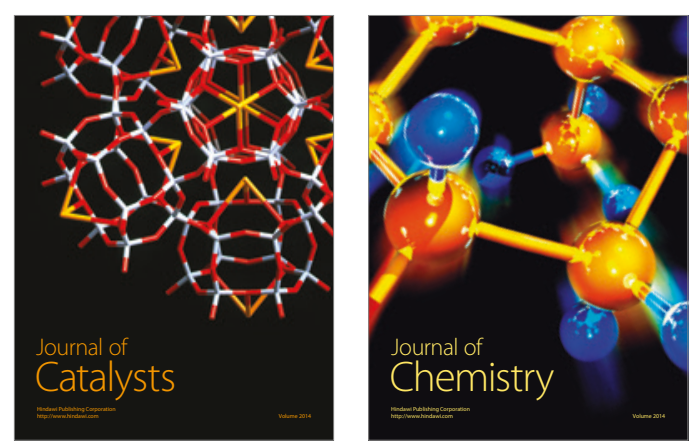
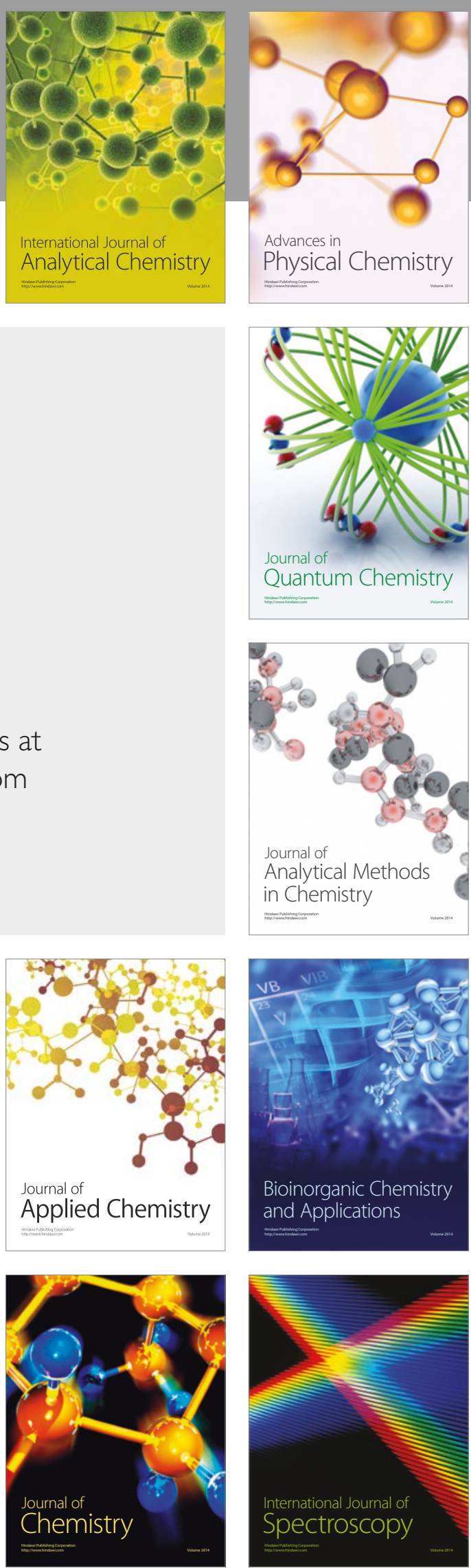